\title{
Preparation and Biological Screening of Novel Heterocyclic Compounds
}

\author{
Pranay Shah, R. I. Patel, P. J. Vyas \\ Sheth M. N. Science College, Patan, Gujarat, India
}

How to cite this paper Pranay Shah | R. I. Patel | P. J. Vyas "Preparation and Biological Screening of Novel Heterocyclic Compounds" Published in International Journal of Trend in Scientific Research and Development (ijtsrd), ISSN: 24566470, Volume-3 | Issue-3, April 2019, pp.632-636, URL: http://www.ijtsrd.co $\mathrm{m} /$ papers/ijtsrd228 15.pdf

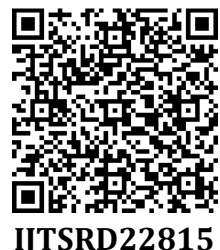

Copyright (C) 2019 by author(s) and International Journal of Trend in Scientific Research and Development Journal. This is an Open Access article distributed under the terms of the Creative Commons

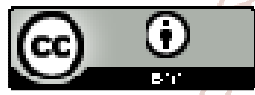
Attribution License (CC BY 4.0) (http://creativecommons.org/licenses/ by/4.0)

\section{INTRODUCTION}

Hydrazones and their heterocyclised products display diverse biological activities including antibacterial, antifungicidal, analgesic, anti-inflammatory properties [110]. These heterocyclic systems find wide use in medicine, agriculture and industry. One of the other compounds says, pyrimidone and their condensed products play a vital role in medicinal chemistry [11-13]. 2-pyrrolidine and its arylidene compounds give good pharmacological properties [14-19]. Hence, it was thought of interest to merge both of pyrrolidine and pyrimidone moieties which may enhance the drug activity of compounds to some extent, or they might possess some of the above mentioned biological activities. From this point of view, the objective of the present work is to prepare new derivatives of pyrimidone containing pyrrolidine moiety. Hence the current communication covers the study of 1-(6-methyl-2-oxo-4-phenyl-1, 2, 3, 4tetrahydropyrimidine-5-carbox amido)-5-oxo-2arylpyrrolidine-3-carboxylic acid (3a-e) followed by Mannich reaction products (4a-e) and imidazolyl derivatives (5a-e). The whole route of synthesis is given in scheme-1.
ABSTRACT

The Pyrimidone derivatives say, N'-arylidine-6-methyl-2-oxo-4-phenyl-1,2,3, 4tetrahydropyrimidonecarbohydrazides (2a-e) were synthesized by condensation of Benzaldehyde derivatives with 6-Methyl-2-oxo-4-phenyl2-oxo-4-phenyl-1,2,3, 4-tetrahydropyrimidone-5-carboxamido)-5-0xo-2-aryl pyrrolidine-3-carboxylic acid (3a-e). These (3a-e) on Mannich reaction with (morpholinomethyl)-5-oxopyrro lidin-1-yl)-6-methyl-2-oxo-4-phenyl-1, 2, 3, 4three series have also been evaluated. The whole synthetic route is shown below.

KEYWORDS: Schiff base, pyrrolidine, Spectral study, antibacterial and antifungal activities. rend in Scientific

Development
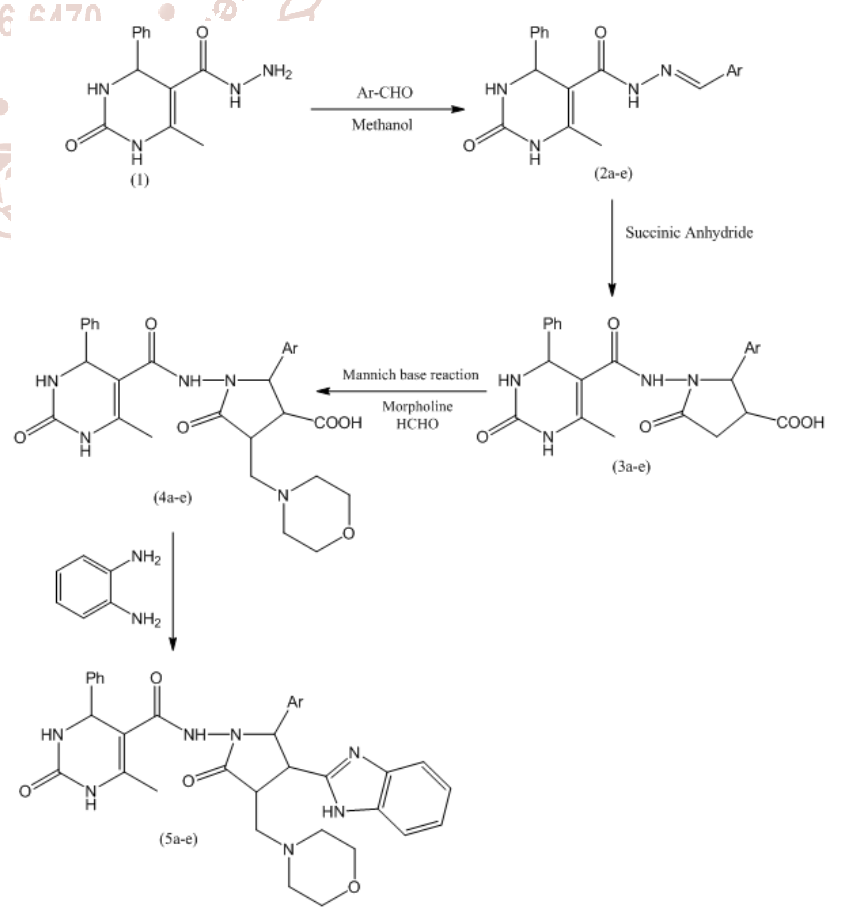

Where $\mathrm{Ar}=(\mathrm{a}) \mathrm{C}_{6} \mathrm{H}_{5}$, (b) $4-\mathrm{CH}_{3}-\mathrm{C}_{6} \mathrm{H}_{4}$, (c) $4-\mathrm{Cl}_{-}-\mathrm{C}_{6} \mathrm{H}_{4}$, (d) $4-\mathrm{Br}-\mathrm{C}_{6} \mathrm{H}_{4}$, (e) $4-\mathrm{OCH}_{3}-\mathrm{C}_{6} \mathrm{H}_{4}$ 


\section{EXPERIMENTAL}

Melting points were determined in open capillary tubes and were uncorrected. The IR spectra were recorded in $\mathrm{KBr}$ pellets on a Nicolet 400D spectrometer and ${ }^{1} \mathrm{H}$ NMR spectra were recorded in DMSO with TMS as internal standard on a Bruker spectrometer at $400 \mathrm{MHz}$. LC-MS of selected samples taken on LC-MSD-Trap-SL_01046. 6-methyl-2-oxo-4-phenyl 1, 2, 3, 4-tetrahydropyrimidine-5-carbohydrazide was prepared by reported method.[20]

Preparation of N'-arylidene-6-methyl-2-oxo-4-phenyl1,2,3,4-tetrahydro pyrimidine-5-carbohydrazide (2ae):- An equimolecular mixture of 6-methyl-2-oxo-4-phenyl1,2,3,4-tetrahydro pyrimidine-5-carbohydrazide (0.01mole) and the aromatic aldehydes [a-e] in ethanol (15ml) was refluxed on a water bath for 2-2.5 hrs. The solid separated was collected by filtration, dried and recrystallized from ethanol. The yields, melting points and other characterization data of these compounds are given in Table -1 .

Preparation of 1-(6-methyl-2-oxo-4-phenyl-1,2,3,4tetrahydropyrimidine-5-carboxamido)-5-oxo-2arylpyrrolidine-3-carboxylic acid (3a-e):- A mixture of N'arylidene-6-methyl-2-oxo-4-phenyl-1,2,3,4-

tetrahydropyrimidine-5-carbo hydrazide (2a-e) (0.01 mole) and Succinic anhydride $(0.01 \mathrm{~mole})$ in p-Xylene $(50 \mathrm{ml})$ was refluxed for 5-7 hrs. The reaction mixture was allowed to stand for 2 days, the solid was filtered. The product thus formed was recrystallized from ethanol to give 1-(6-methyl2-oxo-4-phenyl-1,2,3,4-tetrahydropyrimidine-5carboxamido)-5-oxo-2-arylpyrrolidine-3-carboxylic acid (3ae). The yields, melting points and other characterization data of these compounds are given in Table -2 .

Preparation of 2-aryl-1-(6-methyl-2-oxo-4-phenyl1,2,3,4-tetrahydropyrimidine-5-carboxamido)-4(morpholinomethyl)-5-oxopyrrolidine-3-carboxylic acid (4a-e)[21]:- The above1-(6-methyl-2-oxo-4-phenyl-1,2,3,4tetrahydropyrimidine-5-carboxamido)-5-oxo-2-

arylpyrrolidine-3-carboxylic acid (3a-e) derivatives were reacted with morpholine and $37 \% \mathrm{w} / \mathrm{w}$ formalin at stoichiametric ratio in 1,4-dioxane under refluxed condition for $3 \mathrm{hrs}$.The products were checked by TLC. The yields, melting points and other characterization data of these compounds are given in Table -3 .
Preparation of N-(3-(1H-benzo [d]imidazol-2-yl)-2-aryl4-(morpholinomethyl)-5-oxopyrrolidin-1-yl)-6-methyl2-oxo-4-phenyl-1,2,3,4-tetrahydropyrimidine-5carboxamide (5a-e)[22]:-

A mixture of o-Phenylenediamine and 2-aryl-1-(6-methyl-2oxo-4-phenyl-1,2,3,4-tetrahydropyrimidine-5-

carboxamido)-4-(morpholinomethyl)-5-oxopyrrolidine-3carboxylic acid (4a-e) was refluxed thermally. The reaction mixture was cooled and sodium hydroxide solution was added and then the crude product, $\mathrm{N}-(3-(1 \mathrm{H}-$ benzo[d]imidazol-2-yl)-2-aryl-4-(morpholinomethyl)-5oxopyrrolidin-1-yl)-6-methyl-2-oxo-4-phenyl-1,2,3,4tetrahydropyrimidine-5-carboxamide (5a-e) was washed with ice cold water and dissolved in boiling water for recrystallization, filtered and dried. The yields, melting points and other characterization data of these compounds are given in Table -4 .

\section{RESULTS AND DISCUSSION}

It was observed that 6-methyl-2-oxo-4-phenyl-1,2,3,4tetrahydro pyrimidine-5-carbohydrazide (1) undergoes facile condensation with aromatic aldehydes to afford the corresponding N'-arylidene-6-methyl-2-oxo-4-phenyl1,2,3,4-tetrahydro pyrimidine-5-carbo hydrazide (2a-e). The structures of (2a-e) were confirmed by elemental analysis and IR spectra showing an absorption band at $1630-1660 \mathrm{~cm}^{-}$ $1(\mathrm{C}=\mathrm{N}), 3030-3085 \mathrm{~cm}^{-1}$ (C-H of Ar), $2815-2850 \mathrm{~cm}^{-1}\left(\mathrm{CH}_{3}\right)$, $1720 \mathrm{~cm}^{-1}(\mathrm{CO}), 3260 \mathrm{~cm}^{-1}(-\mathrm{NH}), 735 \mathrm{~cm}^{-1}(\mathrm{C}-\mathrm{Cl}), 590 \mathrm{~cm}^{-1}(\mathrm{C}-$ $\mathrm{Br}), 1235 \mathrm{~cm}^{-1}\left(\mathrm{OCH}_{3}\right) .{ }^{1} \mathrm{H}$ NMR :7.30 $-8.10(10 \mathrm{H}, \mathrm{m}, \mathrm{Ar}-\mathrm{H})$, 8.43-8.80(1H,s, N=CH), 5.63(1H,s,-CH), 6.10-7.92(3H,s,-NH), $2.30\left(3 \mathrm{H}, \mathrm{s}, \mathrm{CH}_{3}\right), 2 \mathrm{~b} ; 2.1\left(3 \mathrm{H}, \mathrm{s}, \mathrm{CH}_{3}\right), 2 \mathrm{e} ; 3.90\left(3 \mathrm{H}, \mathrm{s}, \mathrm{OCH}_{3}\right)$. The $\mathrm{C}$, $\mathrm{H}, \mathrm{N}$ analysis data of all compounds are presented in Table-1.

The structures assigned to 1-(6-methyl-2-oxo-4-phenyl1,2,3,4-tetrahydropyrimidine-5-carboxamido)-5-oxo-2arylpyrrolidine-3-carboxylic acid (3a-e) were supported by the elemental analysis and IR spectra showing an absorption bands at $1720 \mathrm{~cm}^{-1}(\mathrm{C}=0$ of pyrrolidine ring), $3040-3058 \mathrm{~cm}$ ${ }^{1}$ (C-H of Ar), $1660-1670 \mathrm{~cm}^{-1}$ (-CO), $2815-2850 \mathrm{~cm}^{-1}\left(\mathrm{CH}_{3}\right)$, $3260 \mathrm{~cm}^{-1}(-\mathrm{NH}), 735 \mathrm{~cm}^{-1}$ (C-Cl), $590 \mathrm{~cm}^{-1}$ (C-Br), $1235 \mathrm{~cm}^{-1}$ $\left(\mathrm{OCH}_{3}\right)$ for (3a-e) compound. ${ }^{1} \mathrm{H}$ NMR: $7.30-8.10(10 \mathrm{H}, \mathrm{m}$, Ar-H), $4.72\left(1 \mathrm{H}, \mathrm{s}, \mathrm{C}_{2} \mathrm{H}\right.$ of the ring), $3.45\left(1 \mathrm{H}, \mathrm{t}, \mathrm{C}_{3} \mathrm{H}\right), 2.82-$ $2.58\left(2 \mathrm{H}, \mathrm{d}, \mathrm{C}_{4} \mathrm{H}\right), 12.96(1 \mathrm{H}, \mathrm{s},-\mathrm{COOH}), 5.63(1 \mathrm{H}, \mathrm{s},-\mathrm{CH}), 6.10-$ $7.92(3 \mathrm{H}, \mathrm{s},-\mathrm{NH}), 2.30\left(3 \mathrm{H}, \mathrm{s}, \mathrm{CH}_{3}\right), 3 \mathrm{~b} ; 2.1\left(3 \mathrm{H}, \mathrm{s}, \mathrm{CH}_{3}\right), 3 \mathrm{e} ; 3.90$ $\left(3 \mathrm{H}, \mathrm{s}, \mathrm{OCH}_{3}\right)$. The $\mathrm{C}, \mathrm{H}, \mathrm{N}$ analysis data of all compounds are presented in Table- 2 .

Table:-1 Analytical Data and Elemental Analysis of Compounds (2a-e)

\begin{tabular}{|c|c|c|c|c|c|c|c|c|c|}
\hline \multirow{3}{*}{ Compd. } & \multirow{3}{*}{$\begin{array}{c}\text { Molecular formula } \\
\text { (Mol. wt.) }\end{array}$} & \multirow{3}{*}{ Yield } & \multirow{3}{*}{$\begin{array}{c}\text { M.P.* } \\
{ }^{0} \mathrm{C}\end{array}$} & \multicolumn{6}{|c|}{ Elemental Analysis } \\
\hline & & & & \multicolumn{2}{|c|}{$\% \mathrm{C}$} & \multicolumn{2}{|c|}{$\% \mathrm{H}$} & \multicolumn{2}{|c|}{$\% \mathrm{~N}$} \\
\hline & & & & Found & Calcd. & Found & Calcd. & Found & Calcd. \\
\hline $2 \mathrm{a}$ & $\begin{array}{c}\mathrm{C}_{19} \mathrm{H}_{18} \mathrm{~N}_{4} \mathrm{O}_{2} \\
(334)\end{array}$ & 84 & $240-241$ & 68.2 & 68.25 & 5.4 & 5.43 & 16.7 & 16.76 \\
\hline $2 b$ & $\begin{array}{c}\mathrm{C}_{20} \mathrm{H}_{20} \mathrm{~N}_{4} \mathrm{O}_{2} \\
(348)\end{array}$ & 80 & $243-244$ & 68.9 & 68.95 & 5.7 & 5.79 & 16.0 & 16.08 \\
\hline $2 \mathrm{c}$ & $\begin{array}{c}\mathrm{C}_{19} \mathrm{H}_{17} \mathrm{~N}_{4} \mathrm{O}_{2} \mathrm{Cl} \\
(368)\end{array}$ & 82 & $240-242$ & 61.8 & 61.87 & 4.6 & 4.65 & 15.1 & 15.19 \\
\hline $2 d$ & $\begin{array}{c}\mathrm{C}_{19} \mathrm{H}_{18} \mathrm{~N}_{4} \mathrm{O}_{2} \mathrm{Br} \\
(412)\end{array}$ & 83 & $245-247$ & 55.2 & 55.22 & 4.1 & 4.15 & 13.5 & 13.56 \\
\hline $2 \mathrm{e}$ & $\begin{array}{c}\mathrm{C}_{19} \mathrm{H}_{18} \mathrm{~N}_{4} \mathrm{O}_{2} \\
(364)\end{array}$ & 86 & $248-249$ & 65.9 & 65.92 & 5.5 & 5.53 & 15.3 & 15.38 \\
\hline
\end{tabular}

* Uncorrected LC-MS data 2a-351,2d-429 
International Journal of Trend in Scientific Research and Development (IJTSRD) @ www.ijtsrd.com eISSN: 2456-6470

Table:-2 Analytical Data and Elemental Analysis of Compounds (3a-e)

\begin{tabular}{|c|c|c|c|c|c|c|c|c|c|}
\hline \multirow{3}{*}{ Compd. } & \multirow{3}{*}{$\begin{array}{l}\text { Molecular formula } \\
\text { (Mol. wt.) }\end{array}$} & \multirow{3}{*}{ Yield } & \multirow{3}{*}{$\begin{array}{l}\text { M.P.* } \\
{ }^{0} \mathrm{C}\end{array}$} & \multicolumn{6}{|c|}{ Elemental Analysis } \\
\hline & & & & \multicolumn{2}{|c|}{$\% \mathrm{C}$} & \multicolumn{2}{|c|}{$\% \mathrm{H}$} & \multicolumn{2}{|c|}{$\% \mathrm{~N}$} \\
\hline & & & & Found & Calcd. & Found & Calcd. & Found & Calcd. \\
\hline $3 a$ & $\begin{array}{c}\mathrm{C}_{23} \mathrm{H}_{22} \mathrm{~N}_{4} \mathrm{O}_{5} \\
(434)\end{array}$ & 64 & $225-226$ & 63.5 & 63.59 & 5.0 & 5.10 & 12.8 & 12.90 \\
\hline $3 b$ & $\begin{array}{c}\mathrm{C}_{24} \mathrm{H}_{24} \mathrm{~N}_{4} \mathrm{O}_{5} \\
(448)\end{array}$ & 68 & $214-216$ & 64.2 & 64.28 & 5.3 & 5.39 & 12.4 & 12.49 \\
\hline $3 c$ & $\begin{array}{c}\mathrm{C}_{23} \mathrm{H}_{21} \mathrm{ClN}_{4} \mathrm{O}_{5} \\
(468)\end{array}$ & 70 & $212-213$ & 58.9 & 58.91 & 4.5 & 4.51 & 11.9 & 11.95 \\
\hline $3 d$ & $\begin{array}{c}\mathrm{C}_{23} \mathrm{H}_{21} \mathrm{BrN}_{4} \mathrm{O}_{5} \\
(512)\end{array}$ & 72 & $218-219$ & 53.8 & 53.81 & 4.1 & 4.12 & 10.9 & 10.91 \\
\hline $3 e$ & $\begin{array}{c}\mathrm{C}_{24} \mathrm{H}_{24} \mathrm{~N}_{4} \mathrm{O}_{6} \mathrm{~S} \\
(464)\end{array}$ & 67 & $223-225$ & 62.0 & 62.06 & 5.2 & 5.21 & 12.0 & 12.06 \\
\hline
\end{tabular}

The structures assigned to 2-aryl-1-(6-methyl-2-oxo-4-phenyl-1,2,3,4-tetrahydro pyrimidine-5-carboxamido)-4-(morpholino methyl)-5-oxopyrrolidine-3-carboxylic acid (4a-e) were supported by the elemental analysis and IR spectra showing an absorption bands at $1720 \mathrm{~cm}^{-1}\left(\mathrm{C}=0\right.$ of pyrrolidine ring), $3040-3058 \mathrm{~cm}^{-1}\left(\mathrm{C}-\mathrm{H}\right.$ of Ar), $1660-1670 \mathrm{~cm}^{-1}(-\mathrm{CO}), 2815-2850 \mathrm{~cm}^{-1}$ $\left(\mathrm{CH}_{3}, \mathrm{CH}_{2}\right), 3260 \mathrm{~cm}^{-1}(-\mathrm{NH}), 735 \mathrm{~cm}^{-1}(\mathrm{C}-\mathrm{Cl}), 590 \mathrm{~cm}^{-1}(\mathrm{C}-\mathrm{Br}), 1235 \mathrm{~cm}^{-1}\left(\mathrm{OCH}_{3}\right)$ for $(4 \mathrm{a}-\mathrm{e})$ compound. ${ }^{1} \mathrm{H} \mathrm{NMR:} 7.30-8.10(10 \mathrm{H}, \mathrm{m}$, Ar-H), $4.72\left(1 \mathrm{H}, \mathrm{s}, \mathrm{C}_{2} \mathrm{H}\right.$ of the ring), 3.45(1H,t, $\left.\mathrm{C}_{3} \mathrm{H}\right), 2.82\left(1 \mathrm{H}, \mathrm{s}, \mathrm{C}_{4} \mathrm{H}\right), 2.75-2.52\left(2 \mathrm{H}, \mathrm{d}, \mathrm{CH}_{2}\right), 3.66-2.70\left(8 \mathrm{H}, \mathrm{t}, \mathrm{CH}_{2}\right), 12.96(1 \mathrm{H}, \mathrm{s})(\mathrm{COOH})$, $5.63(1 \mathrm{H}, \mathrm{s},-\mathrm{CH}), 6.10-7.92(3 \mathrm{H}, \mathrm{s},-\mathrm{NH}), 2.30\left(3 \mathrm{H}, \mathrm{s}, \mathrm{CH}_{3}\right), 4 \mathrm{~b} ; 2.1\left(3 \mathrm{H}, \mathrm{s}, \mathrm{CH}_{3}\right), 4 \mathrm{e} ; 3.90\left(3 \mathrm{H}, \mathrm{s}, \mathrm{OCH}_{3}\right)$. The $\mathrm{C}, \mathrm{H}, \mathrm{N}$ analysis data of all compounds are presented in Table-3.

Table:-3 Analytical Data and Elemental Analysis of Compounds (4a-e)

\begin{tabular}{|c|c|c|c|c|c|c|c|c|c|}
\hline \multirow{3}{*}{ Compd. } & \multirow{3}{*}{$\begin{array}{l}\text { Molecular formula } \\
\text { (Mol. wt.) }\end{array}$} & \multirow{3}{*}{ Yield } & \multirow{3}{*}{$\begin{array}{l}\text { M.P.* } \\
{ }^{0} \mathrm{C}\end{array}$} & \multicolumn{6}{|c|}{ Elemental Analysis } \\
\hline & & & & \multicolumn{2}{|c|}{$\% \mathrm{C}$} & \multicolumn{2}{|c|}{$\% \mathrm{H}$} & \multicolumn{2}{|c|}{$\% \mathrm{~N}$} \\
\hline & & & & Found & Calcd. & Found & Calcd. & Found & Calcd. \\
\hline $4 a$ & $\begin{array}{c}\mathrm{C}_{28} \mathrm{H}_{31} \mathrm{~N}_{5} \mathrm{O}_{6} \\
(533)\end{array}$ & $71 \circ$ & $232-233$ & 63.0 & 63.03 & 5.8 & 5.86 & 13.1 & 13.13 \\
\hline $4 b$ & $\begin{array}{c}\mathrm{C}_{29} \mathrm{H}_{33} \mathrm{~N}_{5} \mathrm{O}_{6} \\
(547)\end{array}$ & 74 & $236-237$ & 63.6 & 63.61 & 6.0 & 6.07 & 12.7 & 12.79 \\
\hline $4 \mathrm{c}$ & $\begin{array}{c}\mathrm{C}_{28} \mathrm{H}_{30} \mathrm{~N}_{5} \mathrm{O}_{6} \mathrm{Cl} \\
(567)\end{array}$ & 69 & $241-243$ & 59.21 & 59.21 & 5.3 & 5.32 & 12.3 & 12.33 \\
\hline $4 d$ & $\begin{array}{c}\mathrm{C}_{28} \mathrm{H}_{30} \mathrm{~N}_{5} \mathrm{O}_{6} \mathrm{Br} \\
(611)\end{array}$ & 73 & $234-235$ & 54.9 & 54.91 & 4.9 & 4.94 & 11.4 & 11.43 \\
\hline $4 e$ & $\begin{array}{c}\mathrm{C}_{29} \mathrm{H}_{33} \mathrm{~N}_{5} \mathrm{O}_{7} \mathrm{~S} \\
(563)\end{array}$ & 76 & $237-238$ & 61.7 & 61.80 & 5.8 & 5.90 & 12.4 & 12.43 \\
\hline
\end{tabular}

The structures assigned to N-(3-(1H-benzo[d]imidazol-2-yl)-2-aryl-4-(morpholinomethyl)-5-oxopyrrolidin-1-yl)-6-methyl-2oxo-4-phenyl-1,2,3,4-tetrahydropyrimidine-5-carboxamide (5a-e) were supported by the elemental analysis and IR spectra showing an absorption bands at $1720 \mathrm{~cm}^{-1}\left(\mathrm{C}=0\right.$ of pyrrolidine ring), $3040-3058 \mathrm{~cm}^{-1}(\mathrm{C}-\mathrm{H}$, of Ar$), 1660-1670 \mathrm{~cm}^{-1}(-\mathrm{CO}), 3430 \mathrm{~cm}^{-1}$ $(\mathrm{NH}), 2815-2850 \mathrm{~cm}^{-1}\left(\mathrm{CH}_{3}, \mathrm{CH}_{2}\right), 3260 \mathrm{~cm}^{-1}(-\mathrm{NH}), 735 \mathrm{~cm}^{-1}(\mathrm{C}-\mathrm{Cl}), 590 \mathrm{~cm}^{-1}(\mathrm{C}-\mathrm{Br}), 1235 \mathrm{~cm}^{-1}\left(\mathrm{OCH}_{3}\right)$ for (5a-e) compound. ${ }^{1} \mathrm{H}$ NMR: $7.30-8.10(14 \mathrm{H}, \mathrm{m}, \mathrm{Ar}-\mathrm{H}), 4.72\left(1 \mathrm{H}, \mathrm{s}, \mathrm{C}_{2} \mathrm{H}\right.$ of the ring), 3.45(1H,t, $\left.\mathrm{C}_{3} \mathrm{H}\right), 2.82\left(1 \mathrm{H}, \mathrm{s}, \mathrm{C}_{4} \mathrm{H}\right), 2.75-2.52\left(2 \mathrm{H}, \mathrm{d}, \mathrm{CH}_{2}\right), 3.66-$ $2.70\left(8 \mathrm{H}, \mathrm{t}, \mathrm{CH}_{2}\right), 5.63(1 \mathrm{H}, \mathrm{s},-\mathrm{CH}), 5.80-7.92(4 \mathrm{H}, \mathrm{s},-\mathrm{NH}), 2.30\left(3 \mathrm{H}, \mathrm{s}, \mathrm{CH}_{3}\right) 5 \mathrm{~b} ; 2.1\left(3 \mathrm{H}, \mathrm{s}, \mathrm{CH}_{3}\right), 5 \mathrm{e} ; 3.90(3 \mathrm{H}, \mathrm{s}, \mathrm{OCH} 3) . \mathrm{The} \mathrm{C}, \mathrm{H}, \mathrm{N}$ analysis data of all compounds are presented in Table-4.

Table:-4 Analytical Data and Elemental Analysis of Compounds (5a-e)

\begin{tabular}{|c|c|c|c|c|c|c|c|c|c|}
\hline \multirow{3}{*}{ Compd. } & \multirow{3}{*}{$\begin{array}{c}\text { Molecular formula } \\
\text { (Mol. wt.) }\end{array}$} & \multirow{3}{*}{ Yield } & \multirow{3}{*}{$\begin{array}{c}\text { M.P.* } \\
{ }^{0} \mathrm{C}\end{array}$} & \multicolumn{6}{|c|}{ Elemental Analysis } \\
\hline & & & & \multicolumn{2}{|c|}{$\% \mathrm{C}$} & \multicolumn{2}{|c|}{$\% \mathrm{H}$} & \multicolumn{2}{|c|}{$\% \mathrm{~N}$} \\
\hline & & & & Found & Calcd. & Found & Calcd. & Found & Calcd. \\
\hline $5 a$ & $\begin{array}{c}\mathrm{C}_{34} \mathrm{H}_{35} \mathrm{~N}_{7} \mathrm{O}_{4} \\
(605)\end{array}$ & 70 & $241-243$ & 67.4 & 67.42 & 5.8 & 5.82 & 16.1 & 16.19 \\
\hline $5 b$ & $\begin{array}{c}\mathrm{C}_{35} \mathrm{H}_{37} \mathrm{~N}_{7} \mathrm{O}_{4} \\
(619)\end{array}$ & 73 & $255-256$ & 67.8 & 67.83 & 6.0 & 6.02 & 15.8 & 15.82 \\
\hline $5 c$ & $\begin{array}{c}\mathrm{C}_{34} \mathrm{H}_{34} \mathrm{~N}_{7} \mathrm{O}_{4} \mathrm{Cl} \\
(639)\end{array}$ & 76 & $263-265$ & 63.7 & 63.79 & 5.3 & 5.35 & 15.3 & 15.32 \\
\hline $5 d$ & $\begin{array}{c}\mathrm{C}_{34} \mathrm{H}_{34} \mathrm{~N}_{7} \mathrm{O}_{4} \mathrm{Br} \\
(683)\end{array}$ & 77 & $259-260$ & 59.6 & 59.65 & 4.9 & 5.01 & 14.3 & 14.32 \\
\hline $5 e$ & $\begin{array}{c}\mathrm{C}_{35} \mathrm{H}_{37} \mathrm{~N}_{7} \mathrm{O}_{5} \\
(635)\end{array}$ & 72 & $267-268$ & 66.1 & 66.13 & 5.8 & 5.87 & 15.4 & 15.42 \\
\hline
\end{tabular}


The examination of elemental analytical data reveals that the elemental contents are consistence with the predicted structure shown in Scheme-1. The IR data also direct for assignment of the predicted structure. LC-MS data of selected compounds shows the molecular ion peak, which is consistent with their corresponds molecular weight.

\section{BIOLOGICAL SCREENING}

Antibacterial activities

The antibacterial activities of all the compounds were studied against gram-positive bacteria (Staphylococcus aureus and Bacillus subtilis) and gram-negative bacteria (E.coli, and klebsiella promioe) at a concentration of $50 \mu \mathrm{g} / \mathrm{ML}$ by agar cup plate method. A methanol system was used as control in this method. Similar conditions using tetracycline as a control was used standard for comparison. The area of inhibition of zone measured in $\mathrm{mm}$. Compounds $3 \mathrm{c}, 3 \mathrm{e}, 4 \mathrm{c}, 4 \mathrm{e}, 5 \mathrm{c}$ and $5 \mathrm{e}$ were found more toxic for microbes. Other compounds found to be less or moderate active Tables -5 .

Table:-3 Antibacterial Activity of Compounds (3a-e), (4a-e) and (5a-e)

\begin{tabular}{|c|c|c|c|c|}
\hline \multirow{2}{*}{ Compounds } & \multicolumn{2}{|c|}{ Gram +Ve } & \multicolumn{2}{c|}{ Gram -Ve } \\
\cline { 2 - 5 } & Staphylococcus aureus & Bacillus subtilis & E.coli & Klebsiella promioe \\
\hline $3 a$ & 55 & 49 & 62 & 57 \\
\hline $3 b$ & 54 & 54 & 60 & 70 \\
\hline $3 c$ & 57 & 63 & 71 & 59 \\
\hline $3 d$ & 52 & 52 & 62 & 54 \\
\hline $3 \mathrm{e}$ & 58 & 60 & 74 & 74 \\
\hline $4 \mathrm{a}$ & 57 & 53 & 64 & 59 \\
\hline $4 \mathrm{~b}$ & 56 & 58 & 62 & 72 \\
\hline $4 \mathrm{c}$ & 59 & 67 & 73 & 61 \\
\hline $4 \mathrm{~d}$ & 54 & 56 & 63 & 56 \\
\hline $4 \mathrm{e}$ & 59 & 64 & 76 & 75 \\
\hline $5 \mathrm{a}$ & 60 & 54 & 67 & 61 \\
\hline $5 \mathrm{~b}$ & 59 & 59 & 65 & 74 \\
\hline $5 \mathrm{c}$ & 62 & 59 & 76 & 63 \\
\hline $5 \mathrm{~d}$ & 57 & 66 & 65 & 58 \\
\hline $5 \mathrm{e}$ & 63 & & 78 & 76 \\
\hline
\end{tabular}

\section{Antifungal Activities}

The fungicidal activity of all the compounds was studied at $1000 \mathrm{ppm}$ concentration in vitro. Plant pathogenic organisms used were Nigrospora Sp, Aspergillus niger, Botrydepladia thiobromine, and Rhizopus nigricum, Fusarium oxyporium. The antifungal activities of all the compounds (3a-e),(4a-e) and(5a-e) were measured on each of these plant pathogenic strains on a potato dextrose agar (PDA) medium. Such a PDA medium contained potato $200 \mathrm{~g}$, dextrose $20 \mathrm{~g}$, agar $20 \mathrm{~g}$ and water $1 \mathrm{c}$. Five days old cultures were employed. The compounds to be tested were suspended (1000ppm) in a PDA medium and autoclaved at $120^{\circ} \mathrm{C}$ for $15 \mathrm{~min}$. at 15atm. pressure. These media were poured into sterile Petri plates and the organisms were inoculated after cooling the Petri plates. The percentage inhibition for fungi was calculated after five days using the formula given below:

Where,

$\mathrm{X}=$ Area of colony in control plate

$\mathrm{Y}=$ Area of colony in test plate

The fungicidal activity displayed by various compounds (3a-e),(4a-e) and (5a-e) is shown in Tables-6.

Table:-6 Antifungal Activity of Compounds (3a-e), (4a-e) and (5a-e)

\begin{tabular}{|c|c|c|c|c|c|}
\hline \multicolumn{6}{|c|}{ Zone of Inhibition at $1000 \mathrm{ppm}(\%)$} \\
\hline Compounds & Botrydepladia Thiobromine & Rhizopus Nigricum & $\begin{array}{c}\text { Aspergillus } \\
\text { Niger }\end{array}$ & $\begin{array}{c}\text { Nigrospora } \\
\text { Sp. }\end{array}$ & $\begin{array}{l}\text { Fusarium } \\
\text { oxyporium }\end{array}$ \\
\hline $3 a$ & 61 & 62 & 58 & 56 & 66 \\
\hline $3 \mathrm{~b}$ & 59 & 59 & 55 & 60 & 62 \\
\hline $3 \mathrm{c}$ & 73 & 67 & 62 & 72 & 69 \\
\hline $3 \mathrm{~d}$ & 65 & 58 & 59 & 58 & 63 \\
\hline $3 e$ & 70 & 71 & 64 & 65 & 67 \\
\hline $4 a$ & 63 & 64 & 64 & 59 & 67 \\
\hline $4 \mathrm{~b}$ & 75 & 78 & 74 & 73 & 63 \\
\hline $4 \mathrm{c}$ & 76 & 79 & 76 & 76 & 70 \\
\hline $4 \mathrm{~d}$ & 68 & 72 & 72 & 73 & 65 \\
\hline $4 \mathrm{e}$ & 74 & 77 & 79 & 75 & 69 \\
\hline $5 a$ & 64 & 64 & 64 & 59 & 68 \\
\hline $5 b$ & 76 & 78 & 75 & 75 & 65 \\
\hline $5 c$ & 78 & 80 & 77 & 78 & 71 \\
\hline $5 \mathrm{~d}$ & 69 & 73 & 74 & 74 & 66 \\
\hline $5 \mathrm{e}$ & 76 & 79 & 81 & 77 & 70 \\
\hline
\end{tabular}




\section{ACKNOWLEDGEMENT}

The authors are thankful to College management for providing laboratory facilities.

\section{REFERENCES}

[1] E. Gursoy, N. Guzeldemirci-Ulusoy, Eur. J. Med. Chem. $42,2007,320$.

[2] Yu V. Legostacva, Russian J. Org.Chem., 54,2018,146148.

[3] Y. Janin, Bioorg. Med. Chem. 15, 2007, 2479.

[4] M. R. Rao, K. Hart, N. Devanna and K. B. Chandrasekhar, Asian J. Chem. 20,2008, 1402.

[5] A. K. V. Kuar, RRJPPS, 3(1), 2014, 50-54.

[6] A. Nayyar and R. Jain, Curr. Med. Chem. 12, 2006, 1873.

[7] P.Kumar,A.Rai,M.Singh,D.Kumar,A.K. Sahdev and V.Raj, EC Pharmaceutical Sci., 2(3),2016,278-306.

[8] Jan Wu., Ultra Chemistry, 8(2),2012, 269-272.

[9] S. Gemma, G. Kukreja, C. Fattorusso, M. Persico, M. Romano, M. Altarelli, L. Savini, G. Campiani, E. Fattorusso, N. Basilico, Bioorg. Med. Chem. Lett., 16, 2016, 5384.

[10] B. Thomas and J.Harindran, Int. J. Pharm. Sci. and Res., 1(7), 2016, 2795-2804.

[11] İ. Küçükgüzel, Ş.G.Küçükgüzel, S.Rollas, G.Ötük-Sanış, O.Özdemir, İ. Bayrak, T. Altuğ, J.P.Stables, Il Farmaco, 59, 2004, 893-901.
[12] S. Rollas, N. Kalyoncuoglu, D. Sur-Altiner, Y. Yegenoglu, Pharmazie, 48, 1993, 308.

[13] G. Turan-Zitouni, M.F.Sıvacı, S.Kılıç, K.Erol, Molecules, $11,2006,477$.

[14] LQ Al- Mawsawi, R.Dayam, L.Taheri, M.Witvrouw, Z.Debyser, N.Neamati, Bioorg. Med. Chem. Lett. 17(23), 2007, 6472.

[15] C. Plasencia, R. Daym, Q. Wang, J. Pinski, T. R. Jr. Burke, D. I. Quinn and N. Neamati, Mol. Cancer Ther. 4(7), 2005, 1105.

[16] A. Kaur, S. Chaudhri. K. Kumar, M. K. Gupta, K. R. Rawal, Eur. J. Med. Chem., 132, 2017, 108-134.

[17] L. L. Goncalves, L. Devi, L. Rockenbach, G.M. das Neves, L. P. Kagami, R. F. S. Canto, F. Figueiro, A. M. O. Battastini, V. L. Eifler-Lima, Tetrahedron let., 59, 2018, 2759-2762.

[18] M. C. Wa; sch, S. A. Snyder and B. R. Stokwell, Eurr. Opin.Chem.Biol., 14, 2010, 347-361.

[19] A. Bijev, Lett. Drug Des. Discov., 3, 2006, 506.

[20] M. N. Bhoi, M. A. Board, E. A. Pithawala, S. Modi, H. D. Patel, Int. Latters of Chem. Physics and Astronomy, 56, 2015, 82-90.

[21] B. Thomas, L. S. Anju and J. Harindran, Int. J. of Res. in Pharmacy and Chem., 4(2), 2014,351-359.

[22] A. Chawla, R. Kaur and A. Goyal, J. Chem. Pharm. Res., 3(6):2011, 925-944. 